\title{
Tratamiento endovascular de fístula aorto-cava
}

\author{
Endovascular treatment the aorto-caval fistula
}

Miguel O. Villegas ${ }^{1}$, Federico Riolo ${ }^{1}$, Rubén D. Vieira Miño², Marcela Valenzuela ${ }^{3}$, Alberto Pérez Mereles ${ }^{4}$

\section{RESUMEN}

La fístula aorto-cava es una patología muy poco frecuente, que generalmente se da como complicación del aneurisma de aorta abdominal aterosclerótico. Se presenta un paciente con antecedentes de herida de arma de fuego en abdomen, a quien se le realizó rafia en vena cava inferior. Ingreso por fiebre, evolucionó con insuficiencia cardíaca y sospecha de tromboembolismo de pulmón. Se realizó angio-TC que confirma el diagnóstico de tromboembolismo de pulmón y se observó fístula aorto-cava. Se realizó implante de endoprótesis aórtica y embolización de una rama de la arteria hipogástrica. Evolucionó sin insuficiencia cardiaca ni nuevas reintervenciones en el seguimiento a los 3 años.

Palabras claves: fístula aorto-cava, herida de arma de fuego, insuficiencia cardiaca, tratamiento endovascular.

\begin{abstract}
Aorto-caval fistula is a very rare pathology, that usually occurs as a complication of an abdominal aortic aneurysm atherosclerotic. A patient with a history of a gunsho wound in the abdomen is presented, who underwent repair of the inferior vena cava. Then admission for fever, evolved with heart failure and suspected lung thromboembolism. CT scan was performed confirming the diagnosis of lung thromboembolism, aorto-caval fistula was observed. Endovascular treatment was performed with implant of aortic endoprothesis and embolization of the hypogastric artery. Evolved without heart failure or new reinterventions at 3 years follow up.
\end{abstract}

Key words: aorto-caval fistula, gunshot wound, cardiac failure, endovascular treatment.

Revista Argentina de Cardioangiología Intervencionista 2019;10(1):31-34. DOI: 10.30567/RACI/201901/0031-0034

\section{INTRODUCCIÓN}

La presencia de una fístula aorto-cava (FAo-C) es una rara patología que se puede presentar con insuficiencia cardíaca congestiva (ICC), edema de miembros inferiores, congestión venosa. La causa más frecuente es la asociación con aneurisma de aorta abdominal (AAA) aterosclerótico, otras causas menos frecuentes son el aneurisma micótico, lues, desórdenes del tejido conectivo como síndrome de Marfan y síndrome de Ehlers Danlos ${ }^{1}$. La angio-TC es el método diagnóstico por excelencia ${ }^{2}$. Los resultados terapéuticos son difíciles de evaluar dada la baja frecuencia y el tiempo de aparición.

\section{CASO CLÍNICO}

Paciente masculino de 22 años de edad con antecedentes de herida de arma de fuego en abdomen, con perforación de vena cava inferior (VCI) y asas intestinales que fueron reparados quirúrgicamente 2 meses atrás, evolucionó con fiebre, en hemocultivos se aisló Staphylococcus aureus meticilino-resistente, recibió tratamiento antibiótico y fue dado de alta a los 30 días. Ingresó a nuestra institución por dolor abdominal agudo, se realizó laparotomía exploradora presentando líquido de sufrimiento y bridas en asas intestinales que fueron liberadas. Durante la internación evolucionó con disnea en CF IV (NYHA), edemas de miembros

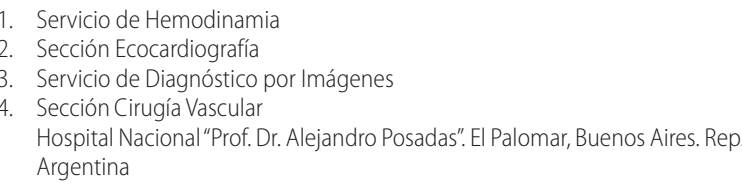

$\triangle$ Correspondencia: Dr. Miquel Osvaldo Villegas. Moreno 827, Campana, Buenos Aires, Argentina. Fax: 011-4469-9206 int.: 1105.miguelovillegas@gmail.com Los autores declaran no tener conflictos de intereses.

Recibido: 11/03/2019| Aceptado: 25/03/2019 inferiores y fiebre, presentó hemocultivos positivos para $S$. aureus meticilino-resistente y se interpretó como ICC en un paciente séptico, se realizó ecocardiograma 2-D que informa dilatación de cavidades derechas y ausencia de vegetaciones.Se realiza angio-TC por sospecha de tromboembolismo de pulmón y la misma informa imágenes compatibles con embolización pulmonar, FAo-C infrarrenal con dilatación de la VCI, fractura de 3er y 4to cuerpos vertebrales lumbares (Figura 1). Ecocardiograma: dilatación de cuatro cavidades, con índice de masa ventricular izquierda aumentada, áreas de ambas aurículas aumentadas, función sistólica del ventrículo derecho conservada, base del ventrículo derecho aumentada, hiperflujo pulmonar, hipertensión pulmonar moderada en reposo, insuficiencia tricuspídea severa, VCI dilatada y sin colapso inspiratorio. Angiografía: presencia de FAo-C infrarrenal (Figura 2A), hipertensión pulmonar severa con presión de la arteria pulmonar de 83/ $34 \mathrm{mmHg}$ (media $50 \mathrm{mmHg}$ ), presión wedge media $30 \mathrm{mmHg}$, presión de ventrículo derecho 80/0-15 $\mathrm{mmHg}$, presión de ventrículo izquierdo $145 / 0-22 \mathrm{mmHg}$, presión de aorta 145/73 $\mathrm{mmHg}$ (media $98 \mathrm{mmHg}$ ).

El cuadro se reinterpreta como pseudoaneurisma micótico entre las vértebras L3 y L4 que produce una FAo-C por dehiscencia de la sutura con embolización pulmonar séptica e ICC.

Se decide realizar tratamiento endovascular con implante de una endoprótesis aórtica Hercules $\mathrm{T}^{\circledast}$ (Microport, Shangai, R.P. China) de 26×26×60 mm (Figura 2B). Una vez implantado se observa llenar la fístula desde una rama de la arteria hipogástrica que es embolizada con coil 3-2-21 (COOK, Bloomington, USA) con resultado exitoso (Figura $2 \mathrm{C}$ a $\mathrm{E})$.

El paciente evoluciona favorablemente. Angio-TC control a los 7 días: aorta abdominal infrarrenal con endoprótesis normoposicionada, sin presencia de endoleak ni llenado venoso por la fístula (Figura 3). Ecocardiograma Doppler a los 7 días: cavidades izquierdas normales. Áreas de ambas aurículas dentro de valores normales. Función sistólica del 

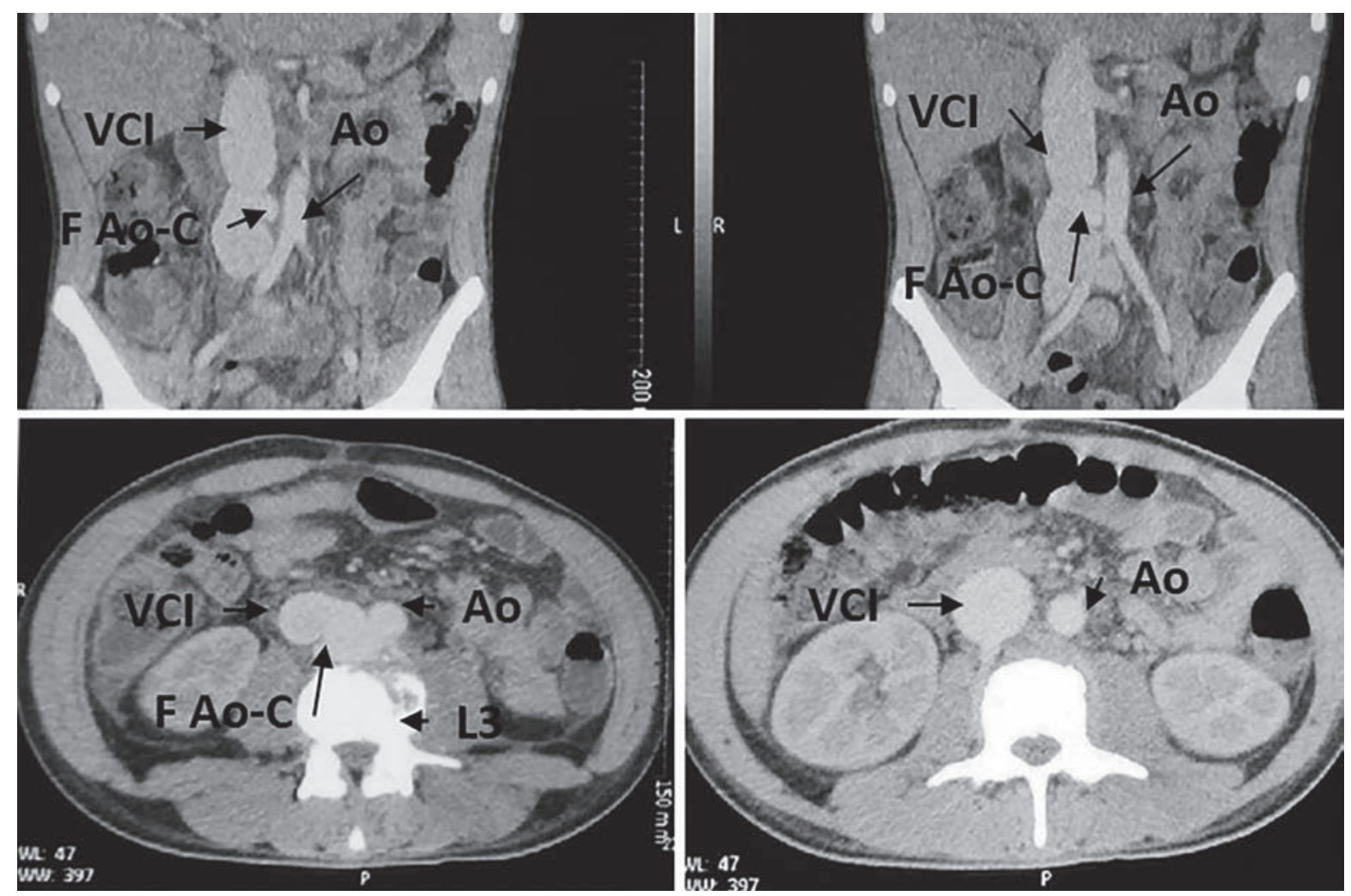

Figura 1. MSTC. Presencia de la fístula aorto-cava (FAo-C), con arteria aorta (Ao) de diámetro conservado y vena cava inferior (VCI) dilatada, 3era vértebra lumbar (L3) con cuerpo fracturado por el proyectil.

ventrículo derecho conservada con actividad hiperdinámica. Reducción de la velocidad del tracto de salida a $94 \mathrm{~cm} / \mathrm{s}$. Insuficiencia tricuspídea menor a la basal. Marcada reducción de la base del ventrículo derecho a $2,46 \mathrm{~cm}$. Colapso inspiratorio y diámetro normal de VCI.

Es dado de alta con tratamiento antibiótico.

Control a los 30 días sin signos de insuficiencia cardiaca ni fiebre. Ecocardiograma Doppler: similar al ecocardiograma control de 7 días.

Control anual durante 3 años sin fiebre ni signos de insuficiencia cardiaca, completó tratamiento antibiótico con TMS durante 6 meses.

\section{DISCUSIÓN}

La FAo-C generalmente se produce por ruptura de un AAA hacia la VCI con una frecuencia del $0.2-6 \%$, no está descripta la frecuencia por otra causa. Las consecuencias fisiopatológicas y hemodinámicas están relacionadas con la aparición súbita del pasaje de sangre desde un sector de alta resistencia, como es el arterial, a uno de bajo flujo como es el circuito venoso. Esto lleva a una reducción de la resistencia periférica, un aumento de la resistencia venosa y del volumen sanguíneo. Por este motivo la frecuencia cardíaca, el gasto cardíaco y el trabajo cardíaco se ven incrementados. Si estos cambios se mantienen el corazón presenta hipertrofia miocárdica, dilatación e insuficiencia cardíaca hiperdinámica. La elevación de la presión venosa renal reduce la presión de perfusión arterial renal activando el sistema renina-angiotensina-aldosterona lo que lleva a una expansión con plasma para aumentar la perfusión, produciendo un círculo vicioso que lleva al paciente a la insuficiencia cardíaca ${ }^{3}$.
La serie de Davidovic et al. mostró en un 48\% signos de shock y el 26\% ICC. El 52\% de los pacientes presentó soplo abdominal continuo, edema de miembros inferiores en el $44 \%$ donde el $8 \%$ estaba asociado a trombosis venosa profunda. El 32\% presentó oliguria, el 20\% hipertensión venosa pélvica manifestada por hematuria y edema escrotal. El comportamiento de la presión venosa central fue elevado en el $62 \%$, en el $34 \%$ era normal y el $4 \%$ estaba baja ${ }^{3}$. Nuestro paciente ingresó por cuadro de ICC y se le auscultaba soplo continuo abdominal.

En un estudio en ratas a las que se les provocó una FAo-C, se evaluó la evolución natural de la ICC, observándose que el $72 \%$ fallecieron en un tiempo medio de 43 semanas, del número total de los fallecidos el $72 \%$ falleció por ICC, el otro $28 \%$ presentó muerte súbita. En la última evaluación previo a la muerte observaron piloerección en el $83 \%$, aumento del diámetro abdominal en el $79 \%$, dificultad respiratoria en el $34 \%$, cianosis en el $10 \%$ y letargia en el 4,2\%. El 28\% falleció sin presentar signos de ICC previo ${ }^{4}$.

En el mismo trabajo de Davidovic et al., el intervalo entre la aparición de la fístula y el tratamiento varía entre 3 horas y 30 días. El 26\% llegó hemodinámicamente inestable y fueron ingresados inmediatamente al quirófano sin estudios diagnósticos previos. El ecocardiograma Doppler se realizó en el $64 \%$, al $24 \%$ se le realizó angiografía convencional y al $40 \%$ angio-TC. En el $40 \%$ no se realizó diagnóstico antes de la cirugía. El diagnóstico correcto está relacionado con la realización de la angiografía convencional o la angio-TC. El 68\% presentaba FAo-C infrarrenal, el resto estaba relacionado a las venas ilíacas o renales. La mortalidad fue del $12 \%$ en el periodo intra- o perioperatorio, en todos es- 


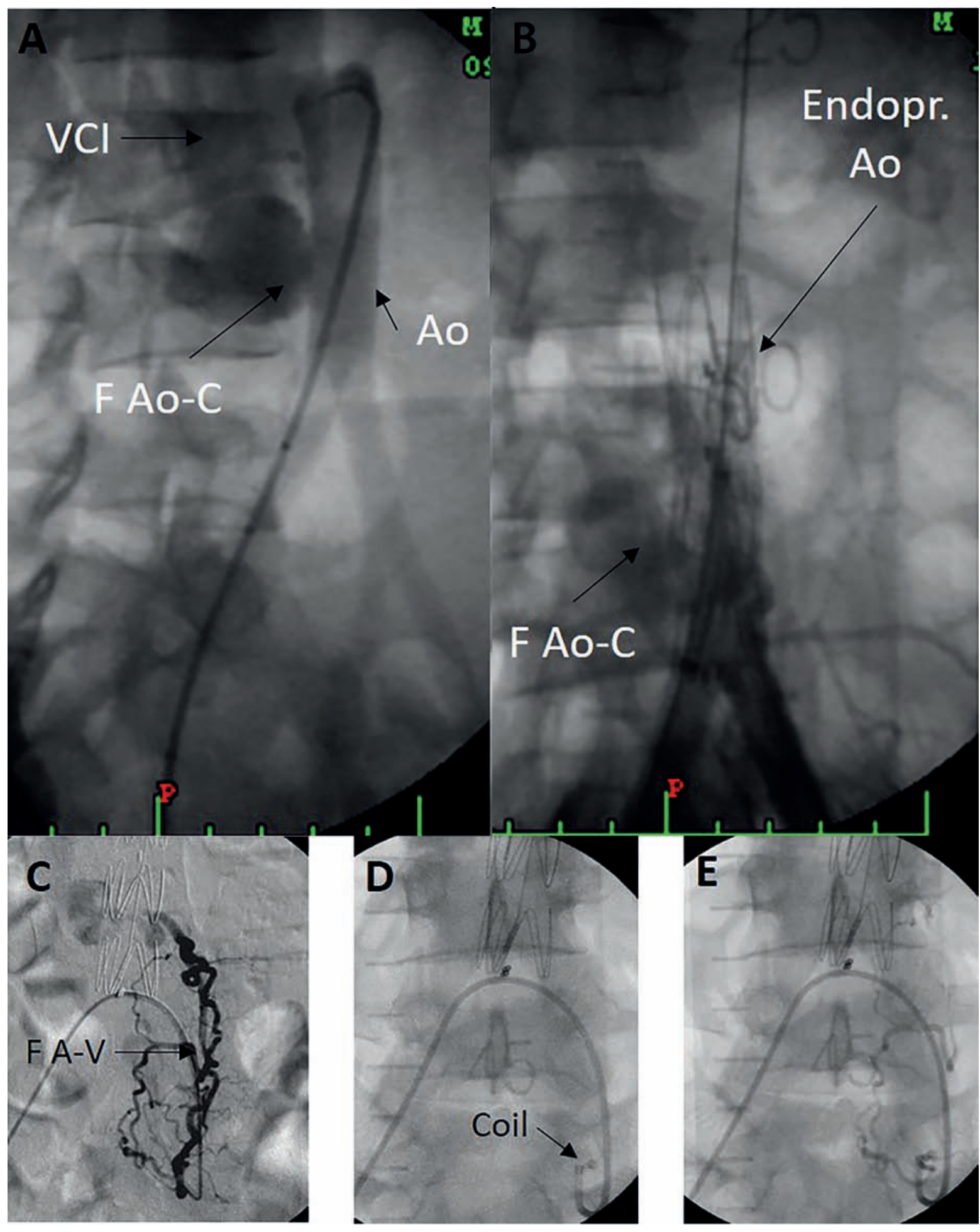

Figura 2. A. Angiografía con inyección desde la aorta donde se observa la fístula aorto-cava (FAo-C) (flecha negra). B. Angiografía control posimplante de endoprótesis HERCULES T๑ (Microport, Shangai, R.P. China) en aorta abdominal (flecha) y llenado de la fístula desde una rama de la arteria hipogástrica (F Ao-C) (flecha). C. Rama de la arteria hipogástrica que llena la fistula (flecha). D. Embolización con coil 25-3-2 (COOK, Bloomington, EE.UU.) sobre la rama (flecha). E. Angiografía control quedando ocluida la fístula.

tos casos el diagnóstico de fístula no se realizó antes de la cirugía. Las causas de muerte fueron sangrado excesivo durante la cirugía, IAM, isquemia colónica y falla multiorgánica ${ }^{3}$.

En presencia de una FAo-C se suele observar un aumento de la presión venosa y signos de embolización pulmonar ${ }^{2}$; nuestro paciente presentó embolización pulmonar diagnosticado por angio-TC.
El tratamiento de la FAo-C en la urgencia generalmente es cierre término-terminal de aorta y venorrafia de la $\mathrm{VCI}^{5}$. El tratamiento también puede ser híbrido, con implante de endoprótesis en VCI y cierre a cielo abierto de la aorta ${ }^{6}$. Nosotros tratamos al paciente con una endoprótesis a nivel arterial y embolización de una rama de la arteria hipogástrica que alimentaba la fístula. 


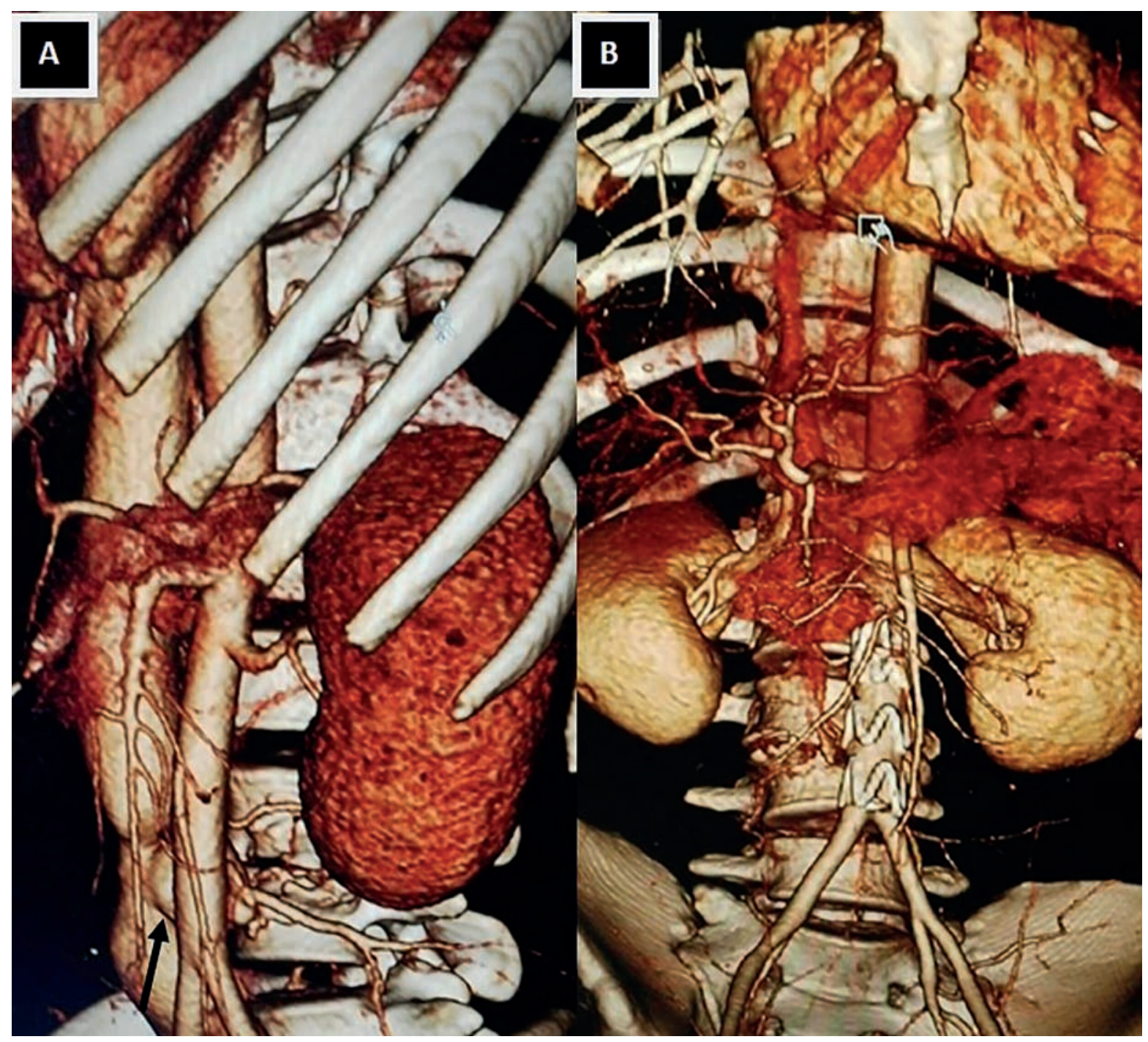

Figura 3. A. Angio-MSTC con imagen de fístula aorto-cava (flecha) y vena cava inferior dilatada. B. En la angio-MSTC se observa endoprótesis en posición aórtica infrarrenal (flecha) sin llenado de la vena cava inferior por tratamiento exitoso de la fistula.

\section{CONCLUSIÓN}

Dada su baja frecuencia, la FAo-C no tiene bien establecido el tratamiento. Nosotros elegimos realizar tra- tamiento endovascular sobre la arteria aorta y embolización de la rama de la hipogástrica con resultado exitoso y seguimiento a los 3 años sin complicaciones ni reintervenciones.

\section{BIBLIOGRAFÍA}

Donatas Gedvilas, Diana Argatu, SauliusLukoševičius, AlgidasBasevičius. Aorto-caval fistula clinically presenting as left renal colic Findings of multislice computed tomography. Medicina (Kaunas) 2008;44(8):619-22.

2. I. Javerliat, M. Coggia, I. Di Centa, P. Alfonsi and O. Goe" au-Brissonnie 're. Undiagnosed Aorto-caval Fistula During Total Laparoscopic Abdominal Aortic Aneurysm Repair: A Cause of Conversion. Eur J VascEndovascSurg2005: Vol 30 (11):497-98.

3. Davidovic Lazar, Drabas Marko, Cvetkovic Slobodan, KosticDusan, Cinarallijas, Banzic Igor. Twenty years of experience in the treatment of spontaneous aorto- venous fistulas in a development country. World J.Surg. 2011 Aug;35(8):1829-34.
4. VojtechMelenovsky, Petra Skaroupkova, Jan Benes, Vera Torresova, Libor Kopkan, LudekCervenka.The Course of Heart Failure Developmentand Mortality in Rats with VolumeOverload due to Aorto-Caval Fistula. KidneyBloodPress Res 2012;35:167-173.

5. Pan George, Bastidas Juan, HasanayiNahid y Floridia Rosario. Bullet embolization from an aorto-caval fistula to the heart. Interactive Cardiovascular and thoracic Surgery 16(2013):710-12

6. Matthias Siepe, Sabrina Koeppe, Wulf Euringer, Christian Schlensak. Aorto-caval fistula from acute rupture of anabdominal aortic aneurysm treated with ahybrid approach J Vasc Surg 2009;49:1574-6. 